\title{
Undulator radiation brightness and coherence near the diffraction limit
}

\author{
Richard P. Walker \\ Diamond Light Source, Oxfordshire OX11 ODE, United Kingdom
}

(Received 2 November 2018; published 28 May 2019)

\begin{abstract}
The brightness and transverse coherence of undulator radiation in synchrotron light sources is investigated using the Wigner formulation in a full 4D treatment. New results are presented on the impact of energy spread on the brightness and coherence as a function of the emittance. In contrast to other work on this topic, it is found that as emittance decreases, the effect of energy spread increases, but as the diffraction limit is approached, the behavior of brightness and coherence diverge. The accuracy of the commonly used Gaussian approximation for brightness and coherence is also explored in detail, resulting in a new conclusion that the commonly used projected source sizes and divergences are not the appropriate quantities to use in the Gaussian approximation to obtain the most accurate results. New expressions are derived for the rms source size and divergence as a function of the energy spread for use in the Gaussian approximation.
\end{abstract}

DOI: 10.1103/PhysRevAccelBeams.22.050704

\section{INTRODUCTION}

A revolution is currently under way in the field of synchrotron light sources with the advent of lower emittance storage rings based on multibend achromat (MBA) lattices [1], which promise synchrotron radiation with a much higher brightness and degree of transverse coherence than previously obtainable. The first of these fourthgeneration storage ring light sources, MAX-IV, recently came into operation in Sweden [2], and this will soon be followed by Sirius in Brazil and the European Synchrotron Radiation Facility upgrade in France. Many other new rings and ring upgrades are at various stages of realization and planning around the globe; for a recent review, see Ref. [3].

These new rings are sometimes referred to as "diffractionlimited storage rings." The diffraction limit is approached when the electron beam emittance $(\varepsilon)$ is reduced to the point that it becomes comparable to the intrinsic photon beam emittance. This is, however, an imprecise term, since the diffraction limit is wavelength dependent: it is most usually taken to mean $\varepsilon \sim \lambda / 4 \pi$, where $\lambda$ is the radiation wavelength. Despite the fact that all current third-generation storage rings already operate near the diffraction limit at longer wavelengths, as well as at X-ray wavelengths in the vertical plane, the recent development of low emittance MBA lattices has reopened questions about what exactly constitutes the "diffraction limit" and how close one can approach it.

Published by the American Physical Society under the terms of the Creative Commons Attribution 4.0 International license. Further distribution of this work must maintain attribution to the author(s) and the published article's title, journal citation, and DOI.
In particular, as the emittance becomes very small, it becomes increasingly important to understand the influence of the energy spread of the electron beam [4], which has been ignored in many of the early studies of brightness and coherence.

The aim of this paper is to investigate how closely one can approach the diffraction limit when the effects of electron beam emittance and energy spread are included and also to explore the validity of the commonly used Gaussian approximation (GA). We will also shed further light on the recently highlighted "paradox" that energy spread by itself does not influence the peak brightness but does degrade the transverse coherence off axis [4].

\section{THE GAUSSIAN APPROXIMATION FOR BRIGHTNESS AND COHERENCE}

The peak brightness of the radiation emitted by an undulator is commonly estimated by the following formula, known as the Gaussian approximation:

$$
\mathcal{B}_{o}=\frac{F}{4 \pi^{2} \Sigma_{x} \Sigma_{x^{\prime}} \Sigma_{y} \Sigma_{y^{\prime}}},
$$

where $F$ is the spectral flux (photons per second per $0.1 \%$ bandwidth) and $\Sigma_{x, y}$ and $\Sigma_{x^{\prime}, y^{\prime}}$ are the rms beam sizes and divergences, respectively, in the horizontal $(x)$ and vertical $(y)$ planes:

$$
\begin{aligned}
& \Sigma_{x}=\left(\sigma_{x}^{2}+\sigma_{R}^{2}\right)^{1 / 2}, \quad \Sigma_{y}=\left(\sigma_{y}^{2}+\sigma_{R}^{2}\right)^{1 / 2}, \\
& \Sigma_{x^{\prime}}=\left(\sigma_{x \prime}^{2}+\sigma_{R^{\prime}}^{2}\right)^{1 / 2}, \quad \Sigma_{y^{\prime}}=\left(\sigma_{y \prime}^{2}+\sigma_{R^{\prime}}^{2}\right)^{1 / 2},
\end{aligned}
$$

which incorporate electron $\left(\sigma_{x}, \sigma_{x^{\prime}}, \sigma_{y}, \sigma_{y^{\prime}}\right)$ and intrinsic photon dimensions $\left(\sigma_{R}, \sigma_{R^{\prime}}\right)$. Equation (1) was first 
introduced in the context of undulators by Krinsky [5]; his expression for $\sigma_{R}$ has, however, been superseded by a more correct treatment of diffraction effects introduced in Refs. [6,7].

The photon emittance $\left(\varepsilon_{R}=\sigma_{R} \sigma_{R^{\prime}}\right)$ entering in Eq. (1b) is usually assumed to be $\lambda / 4 \pi$, since this is the appropriate value for the Gaussian mode of an optical resonator [6]. In the limit of zero electron beam emittance, we have therefore $\mathcal{B}_{o}=F /(\lambda / 2)^{2}$ and, since a Gaussian mode is fully coherent,

$$
F_{\text {coh }}=\mathcal{B}_{o}\left(\frac{\lambda}{2}\right)^{2}
$$

It then follows directly from Eqs. (1) and (2) that the degree of spectral coherence (ratio of coherent flux to total flux) is given by

$$
\zeta=\frac{F_{\mathrm{coh}}}{F}=\frac{(\lambda / 4 \pi)^{2}}{\Sigma_{x} \Sigma_{x^{\prime}} \Sigma_{y} \Sigma_{y^{\prime}}} .
$$

Stated in this way, these are, however, only reasonable propositions but not fundamental results. Equation (1) is valid for a Gaussian mode but is not necessarily true for undulator radiation. Equations (2) and (3) follow from Eq. (1) at zero emittance but are not necessarily true, in general. The simplicity of these expressions has, however, guaranteed their widespread use, although not without some difficulties.

The convolution of the photon and electron dimensions expressed in the above formulas is based on both photons and electrons having Gaussian distributions. While this is a good approximation for the electron beam, at least in storage rings which are being considered here, it is not for the photons, and this has led to a range of different formulas being put forward. Table I lists various definitions of the radiation parameters that are currently in use, together with the corresponding radiation emittance $\left(\varepsilon_{R}=\sigma_{R} \sigma_{R^{\prime}}\right)$ and beta values $\left(\beta_{R}=\sigma_{R} / \sigma_{R^{\prime}}\right)$. The significance of the beta value is that in the Gaussian approximation, Eq. (1), the optimum brightness is obtained when the electron beam beta values $\left(\beta_{x, y}=\sigma_{x, y} / \sigma_{x^{\prime}, y^{\prime}}\right)$ equal the radiation beta value; i.e., the radiation and electron phase-space ellipses are "matched."

The difference between the various formulas is clearly important if one is attempting to optimize the brightness and approach the diffraction limit. In addition to this, model 3, in

TABLE I. Various definitions of intrinsic undulator radiation parameters.

\begin{tabular}{lccccc}
\hline \hline Model & $\sigma_{R^{\prime}}$ & $\sigma_{R}$ & $\varepsilon_{R}$ & $\beta_{R}$ & Reference \\
\hline 1 & $\sqrt{\lambda / L}$ & $\sqrt{\lambda L} / 4 \pi$ & $\lambda / 4 \pi$ & $L / 4 \pi$ & {$[6]$} \\
2 & $\sqrt{\lambda / 2 L}$ & $\sqrt{2 \lambda L} / 4 \pi$ & $\lambda / 4 \pi$ & $L / 2 \pi$ & {$[7,8]$} \\
3 & $\sqrt{\lambda / 2 L}$ & $\sqrt{2 \lambda L} / 2 \pi$ & $\lambda / 2 \pi$ & $L / \pi$ & {$[9,10]$} \\
4 & $\sqrt{\lambda / 4 L}$ & $\sqrt{\lambda L} / 2 \pi$ & $\lambda / 4 \pi$ & $L / \pi$ & {$[11]$} \\
\hline \hline
\end{tabular}

particular, has caused some conceptual difficulties. Since the emittance in this case is $\lambda / 2 \pi$, Eq. (3) would give (in the limit of zero emittance) a degree of coherence of $1 / 4$, whereas the single-electron radiation must by definition have full transverse coherence. The reason why different models for the source size and divergence have been put forward is that the radiation phase space is not at all Gaussian in nature. The contradiction that arises with model 3 is that, as we will see later, it is based on projected phase-space widths, which are not the appropriate quantities for calculating the brightness and degree of coherence.

\section{BRIGHTNESS AND COHERENCE BASED ON THE WIGNER FUNCTION}

The now widely accepted definition of the brightness distribution in phase space is based on the Wigner distribution $[4,6-8,10-14]$ :

$$
\begin{aligned}
\mathcal{B}\left(x, x^{\prime}, y, y^{\prime}\right)= & \frac{2 \varepsilon_{o} c}{h} \frac{1}{\lambda^{2}} \frac{I}{e} \int_{-\infty}^{\infty} \int_{-\infty}^{\infty} \bar{E}\left(x^{\prime}+\frac{\theta_{x}}{2}, y^{\prime}+\frac{\theta_{y}}{2}\right) \\
& \times \bar{E}^{*}\left(x^{\prime}-\frac{\theta_{x}}{2}, y^{\prime}-\frac{\theta_{y}}{2}\right) \\
& \times \exp \left(-i \frac{2 \pi}{\lambda}\left(x \theta_{x}+y \theta_{y}\right)\right) d \theta_{x} d \theta_{y},
\end{aligned}
$$

where $\bar{E}$ is the electric field in its angular representation and $\bar{E}^{*}$ its complex conjugate; $I$ is the electron beam current. From the brightness function, we will calculate the peak, i.e., central, brightness $\mathcal{B}_{o}=\mathcal{B}(0,0,0,0)$, as well as the average brightness defined in Ref. [12]:

$$
\mathcal{B}_{\mathrm{av}}=\frac{\iiint \int \mathcal{B}^{2}\left(x, x^{\prime}, y, y^{\prime}\right) d x d x^{\prime} d y d y^{\prime}}{F},
$$

where $F=\iiint \int\left(\mathcal{B}\left(x, x^{\prime}, y, y^{\prime}\right) d x d x^{\prime} d y d y^{\prime}\right.$ is the total flux. In the following, all integrals are implicitly taken from $-\infty$ to $+\infty$. The average brightness is directly related to the overall degree of spectral coherence, which is defined as follows [12]:

$\zeta=\lambda^{2} \frac{\iiint \int \mathcal{B}^{2}\left(x, x^{\prime}, y, y^{\prime}\right) d x d x^{\prime} d y d y^{\prime}}{F^{2}}=\lambda^{2} \frac{\mathcal{B}_{\mathrm{av}}}{F}$.

We will now use these definitions to put the previous discussion about brightness and coherence on a firmer footing. It can be shown analytically directly from Eq. (4) that for any symmetric electric field distribution, and therefore for single-electron undulator radiation (i.e., with zero emittance and energy spread) at any value of detuning $[8,10,12]$,

$$
\mathcal{B}_{o}=\frac{F}{(\lambda / 2)^{2}}
$$


This is the same as Eq. (2); however, this has now been derived, in general, from the Wigner formula for brightness. Consistency with Eq. (1) in the limit of zero emittance then demands that the photon emittance has a value of $\varepsilon_{R}=\lambda / 4 \pi$. In other words, for Eq. (1) to give the same brightness as that of the Wigner formulation in the case of zero emittance undulator radiation, the photon emittance must have this value.

We can also examine analytically the extreme case of a true Gaussian distribution of brightness, consistent with Eq. (1):

$$
\begin{aligned}
\mathcal{B}= & \mathcal{B}_{o} \exp \left(-x^{2} / 2 \Sigma_{x}^{2}\right) \exp \left(-x^{\prime 2} / 2 \Sigma_{x^{\prime}}^{2}\right) \\
& \times \exp \left(-y^{2} / 2 \Sigma_{y}^{2}\right) \exp \left(-y^{\prime 2} / 2 \Sigma_{y^{\prime}}^{2}\right) .
\end{aligned}
$$

Equations (5) and (6) can then be easily evaluated, and we obtain directly

$$
\mathcal{B}_{\mathrm{av}}=\frac{\mathcal{B}_{o}}{4}
$$

and $\zeta=(\lambda / 4 \pi)^{2} / \Sigma_{x} \Sigma_{x^{\prime}} \Sigma_{y} \Sigma_{y^{\prime}}$. The use of the definitions of brightness and degree of coherence based on the Wigner formalism therefore provides a firm basis for the approximations introduced in the previous section, having shown that they are valid for undulator radiation not only in the case of zero electron beam emittance, but also in the emittance-dominated regime, where the electron beam is well represented by Gaussian distributions. Having fixed the photon emittance $\varepsilon_{R}=\lambda / 4 \pi$, we will answer the question of what is the appropriate beta value in the next section.

\section{SINGLE-ELECTRON EMISSION}

We will restrict ourselves to the horizontally polarized component of radiation generated close to the axis in a standard planar undulator with a vertical magnetic field component, and we will further restrict ourselves to loworder odd harmonics, allowing us to simplify Eq. (4) as follows:

$$
\begin{aligned}
\mathcal{B}\left(x, x^{\prime}, y, y^{\prime}\right)= & \frac{\alpha \gamma^{2} N^{2} F_{n}(K)}{\lambda^{2}} \frac{I}{e} \int_{-\infty}^{\infty} \int_{-\infty}^{\infty} E\left(x^{\prime}+\frac{\theta_{x}}{2}, y^{\prime}+\frac{\theta_{y}}{2}\right) E\left(x^{\prime}-\frac{\theta_{x}}{2}, y^{\prime}-\frac{\theta_{y}}{2}\right) \\
& \times \exp \left(-i \frac{2 \pi}{\lambda}\left(x \theta_{x}+y \theta_{y}\right)\right) d \theta_{x} d \theta_{y},
\end{aligned}
$$

in which $\alpha$ is the fine structure constant, $\gamma$ is the relativistic factor, $N$ is the number of undulator periods, and $F_{n}(K)$ is the usual undulator flux function for the $n$th harmonic, $K$ being the deflection parameter [10]:

$$
\begin{aligned}
F_{n}(K)= & \frac{n^{2} K^{2}}{\left(1+K^{2} / 2\right)^{2}}\left\{J_{\frac{n-1}{2}}\left[\frac{n K^{2}}{4\left(1+K^{2} / 2\right)}\right]\right. \\
& \left.-J_{\frac{n+1}{2}}\left[\frac{n K^{2}}{4\left(1+K^{2} / 2\right)}\right]\right\}^{2} .
\end{aligned}
$$

$E$ is now the line-shape function:

$$
E(\theta)=\frac{\sin \left(N \pi \omega / \omega_{1}\right)}{\left(N \pi \omega / \omega_{1}\right)},
$$

where $\omega_{1}=4 \pi c \gamma^{2} / \lambda_{o}\left(1+K^{2} / 2\right)$ is the fundamental on-axis emission frequency. For a given difference between the frequency of interest and the on-axis frequency, i.e., the "detuning" $N \Delta \omega / \omega_{1}$, and relative electron energy deviation $\delta=\Delta \gamma / \gamma$, the argument of the line-shape function can be written as follows:

$$
\frac{N \omega}{\omega_{1}}=\frac{\theta^{2}}{2 \lambda / L}+N\left(\frac{\Delta \omega}{\omega_{1}}\right)-2 n N \delta .
$$

In this formulation, the line-shape function fully describes the radiation emission; i.e., we ignore the additional interference effects from within a single period [15] which would require a more complex computation. We use this simplification, valid for low-order harmonics, specifically in order to be able to calculate the full 4D Wigner distribution including the energy spread and emittance, which is computationally very intensive.

For the following calculations, we take as an example an undulator [16] which is a potential candidate for the proposed ultralow emittance PETRA IV ring [17]. The parameters for these and all subsequent calculations are given Table II. Figure 1 shows sections of the brightness distribution in the $x-x^{\prime}$ (or, equally, the $y-y^{\prime}$ ) plane, at three different detunings. In these and subsequent figures, the $x$ and $x^{\prime}$ axes have been normalized to the rms values of the projected radiation size and divergence, i.e., model 3 in Table I. It is very evident that the brightness is nonGaussian and, furthermore, is not separable into functions of each phase-space coordinate, meaning that any attempt to approximate it with Gaussian distribution will inevitably be limited in accuracy. 

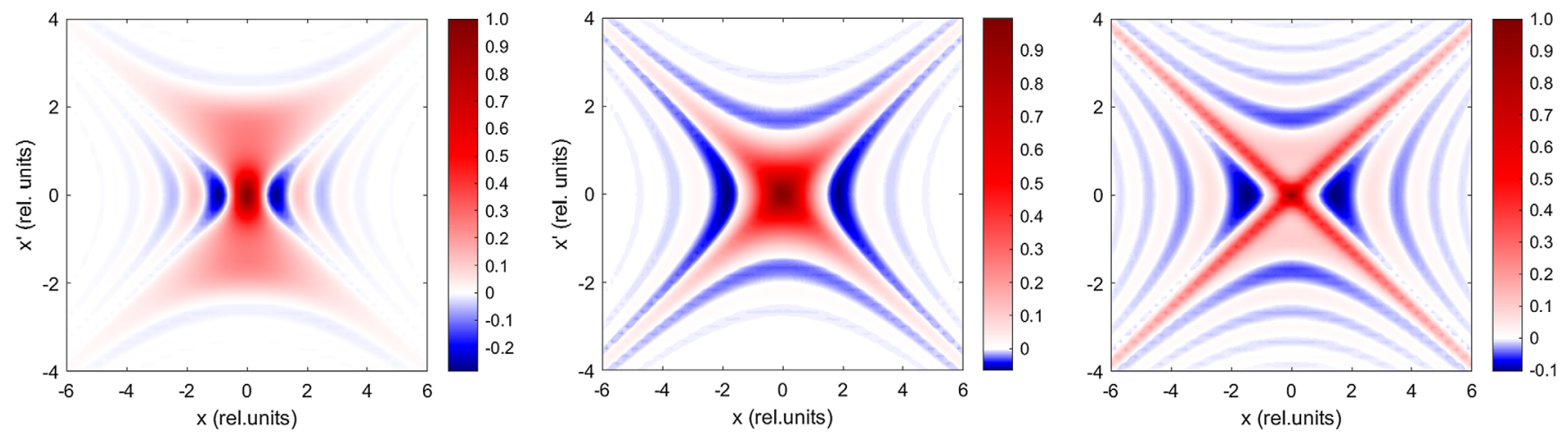

FIG. 1. Brightness distributions at various detunings, $N \Delta \omega / \omega_{1}=-1$ (left), 0 (center), and +1 (right), normalized to unity in each case.

Figure 2 shows the calculated peak brightness, average brightness, and total flux as a function of the detuning. Here and in the following, we use the standard units for flux (photons per second per $0.1 \%$ bandwidth) and brightness (flux per $\mathrm{mm}^{2}$ per $\mathrm{mrad}^{2}$ ). It can be seen that both brightness curves follow the well-known integrated flux vs detuning curve. The results confirm Eqs. (7) and (8) and also that the degree of coherence is indeed unity. These results are different from those presented in Ref. [12], in which 2D projections of the Wigner distribution are calculated. As the author of Ref. [12] points out, because the radiation field is nonseparable, this leads to incorrect results for the average brightness and degree of coherence in a $2 \mathrm{D}$ projection. For this reason, the full $4 \mathrm{D}$ formulation will be used throughout this paper.

To be able to use the Gaussian approximations, Eqs. (1) and (3), to estimate brightness and coherence, we need to be able to calculate appropriate source sizes and divergences. There are, however, many different ways of defining source size and divergence. One method is to take a 1D section through the brightness distribution and then estimate the source size as that of a Gaussian function having the same maximum value and integral, i.e.,

$$
\Sigma_{x}=\frac{\int \mathcal{B}(x, 0,0,0) d x}{\sqrt{2 \pi} \mathcal{B}(x, 0,0,0)_{\max }},
$$

and similarly for the other dimensions. For want of better terminology, we will refer to these as "core" rms values and

TABLE II. Electron beam and undulator parameters used for the simulations.

\begin{tabular}{lc}
\hline \hline Electron energy & $6 \mathrm{GeV}$ \\
Beam current $(I)$ & $0.1 \mathrm{~A}$ \\
Period length $\left(\lambda_{o}\right)$ & $0.018 \mathrm{~m}$ \\
Number of periods $(N)$ & 220 \\
Length $(L)$ & $3.96 \mathrm{~m}$ \\
Deflection parameter $(K)$ & 1.341 \\
Photon energy & $10 \mathrm{keV}$ \\
Harmonic number $(n)$ & 1 \\
\hline \hline
\end{tabular}

the product of them as the "core emittance." Another method is to take the 2D-projected spatial and angular intensities, $d F / d S=\iint \mathcal{B}\left(x, x^{\prime}, y, y^{\prime}\right) d x^{\prime} d y^{\prime}$ and $d F / d \Omega=$ $\iint \mathcal{B}\left(x, x^{\prime}, y, y^{\prime}\right) d x d y$, respectively, and "fit" a twodimensional Gaussian function having the same integral and peak value, i.e.,

$$
\begin{aligned}
\Sigma_{x, y} & =\left(\frac{F}{2 \pi(d F / d S)_{\max }}\right)^{1 / 2} \text { and } \\
\Sigma_{x^{\prime}, y^{\prime}} & =\left(\frac{F}{2 \pi(d F / d \Omega)_{\max }}\right)^{1 / 2} .
\end{aligned}
$$

Such a method can, however, be applied only when there is circular symmetry. Yet another method is to calculate the full 3D-projected intensity distributions along the axes of interest, e.g., $d F / d x=\iiint \mathcal{B} d x^{\prime} d y^{\prime} d y$ and $d F / d x^{\prime}=$ $\iiint \mathcal{B} d x d y d y^{\prime}$, and then fit one-dimensional Gaussian functions. In general, these methods yield different values for the effective rms widths, whereas in the case of a true Gaussian brightness distribution they would all be the same. Figure 3 shows the three distributions, together with

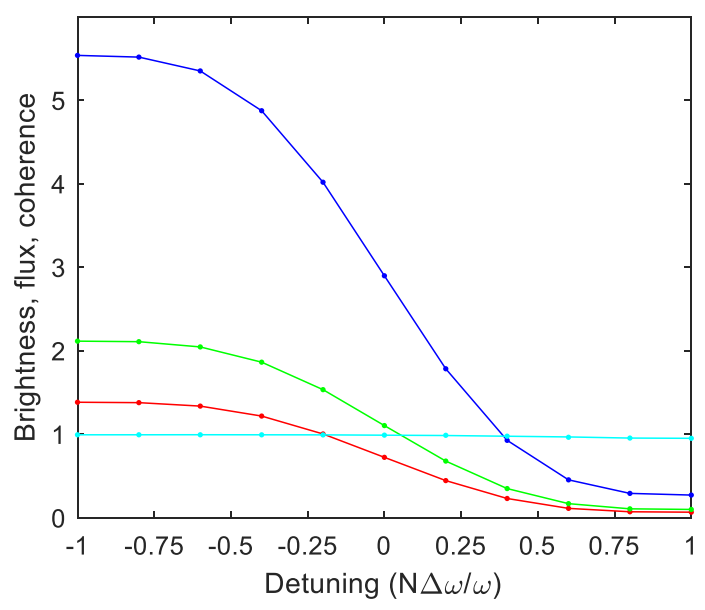

FIG. 2. Peak brightness $\left(\times 10^{23}\right.$, blue line $)$, average brightness $\left(\times 10^{23}\right.$, red line $)$, total flux $\left(\times 10^{15}\right.$, green line $)$, and degree of coherence (cyan line) as a function of the detuning. 

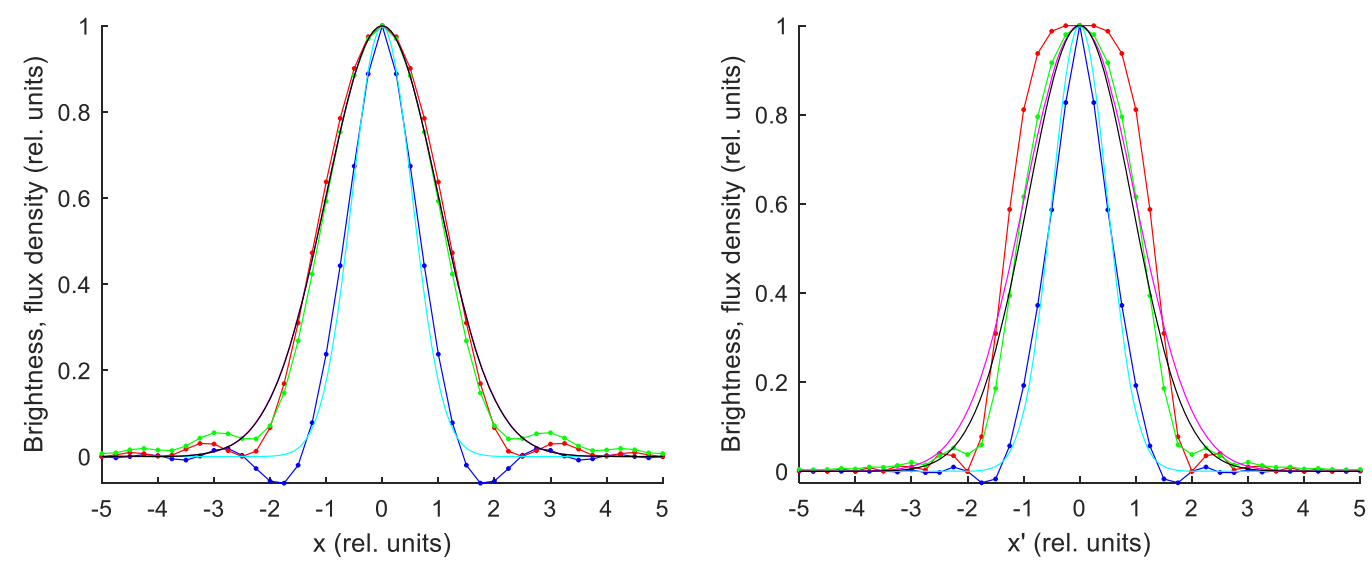

FIG. 3. Spatial (left) and angular (right) distributions of the brightness function; 1D sections (blue line), sections of the 2D projected flux density (red line), and full 3D projections (green line) along the respective axes, with respective Gaussian fits (cyan, magenta, and black lines); magenta and black curves are very close together.

their respective Gaussian fits, for the case of zero detuning. The 1D sections are well fitted by Gaussians with rms values of very close to 0.5 (in relative units), whereas the two projections (despite being different in shape as a

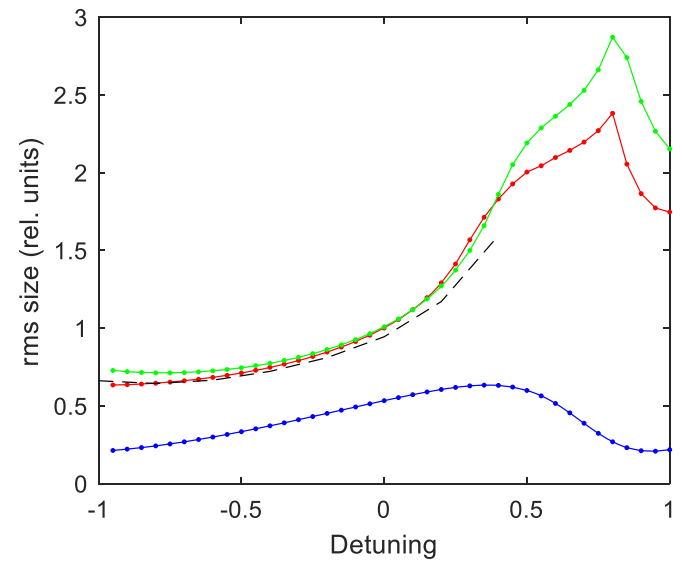

function of the angle) are both reasonably well fitted by Gaussians with rms values of close to 1.0. The core emittance is therefore $\lambda / 8 \pi$, in agreement with Ref. [12], whereas the projections give an emittance of $\lambda / 2 \pi$. Thus,

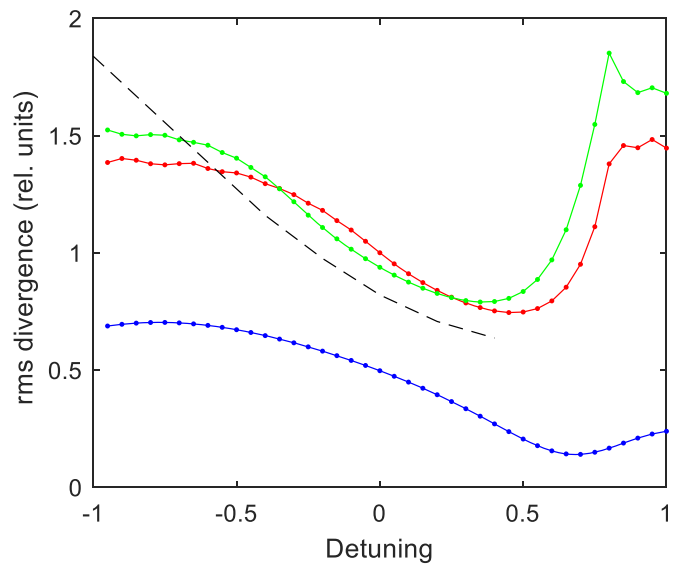

FIG. 4. rms beam sizes (left) and divergences (right) in relative units as a function of the detuning obtained from 1D sections (blue line), 2D projections (red line), and full 3D projections (green line). The dashed black curves are from Ref. [18].
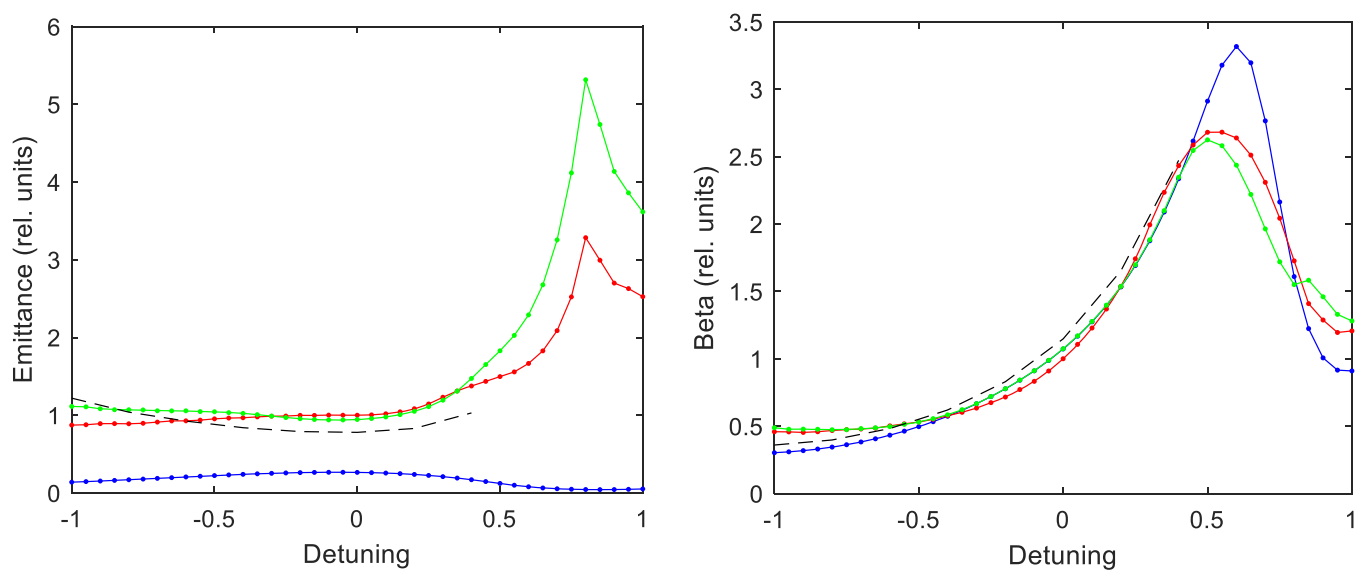

FIG. 5. Data from Fig. 4 expressed as emittance (left) and beta values (right) in relative units. 
none of these methods of describing the phase-space widths agree with the Gaussian approximation, which requires an emittance of $\lambda / 4 \pi$. In other words, the phase-space widths which give the correct brightness and coherence using Eqs. (1) and (3) lie in between those obtained by 1D sections or $2 \mathrm{D}$ or $3 \mathrm{D}$ projections. Interestingly though, the respective beta functions are in all three cases very close to unity, in relative units, i.e., $\beta_{R}=L / \pi$. We conclude, therefore, that model 4 in Table I is the correct one to describe the basic single-electron emission at zero detuning, and in the following the term "Gaussian approximation" will implicitly assume this.

We now examine the phase-space widths as a function of the detuning shown in Fig. 4. The rms values of the projected profiles are in reasonable agreement with the results of Ref. [18]; however, the rms divergence values deviate, because the angular profile is strongly nonGaussian, and Ref. [18] employs a different way of determining the equivalent rms value.

The same data expressed as emittances and beta values are shown in Fig. 5. The emittance remains roughly constant towards negative detuning as required (although not with the required value of $\lambda / 4 \pi, 0.5$ in relative units). At positive detuning, there is a rapid increase in the emittance and beta value. In this region, the reduced intensity and the fact that the phase-space distribution becomes more oscillatory (see Fig. 1) make the problem computationally more difficult, requiring attention to the parameters chosen for the range of integration.

In conclusion, the brightness distribution of undulator radiation is complex and not well represented by a product of Gaussian functions. Even at zero detuning, the phasespace widths required to match the Gaussian approximation do not correspond with any obvious measure of width, and the agreement deviates even further at positive detuning. Nevertheless, given the simplicity of Eqs. (1) and (3) and their widespread use in making approximate calculations of brightness and coherence, we will continue to investigate to what extent they can be used when incorporating also the effects of energy spread and emittance in the following sections, concentrating on the case of zero detuning.

\section{EFFECT OF ELECTRON BEAM ENERGY SPREAD}

The effect of energy spread can be included by integrating the brightness function over the energy distribution:

$$
\mathcal{B}\left(x, x^{\prime}, y, y^{\prime}\right)=\int \mathcal{B}\left(x, x^{\prime}, y, y^{\prime}, \delta\right) P(\delta) d \delta
$$

where the relative energy deviation $\delta=\Delta E / E$ is assumed to have a Gaussian probability distribution $P(\delta)=$ $\exp -\left(\delta^{2} / 2 \sigma_{E}^{2}\right) / \sqrt{2 \pi} \sigma_{E}$.

It is evident directly from Eq. (10) that the effect of an energy deviation can be quite large. For example, with a typical $0.1 \% \mathrm{rms}$ energy spread in the undulator of Table II, there is an rms detuning of 0.44 , which is not insignificant given the effects of detuning shown in Figs. 4 and 5. Figure 6 (left) shows the $x-x^{\prime}$ phase space with $0.1 \%$ rms energy spread, and this confirms the significant "smearing out" of the brightness distribution, particularly evident in the $x^{\prime}$ direction.

Figure 7 shows the peak brightness, average brightness, and degree of coherence as a function of the energy spread. The central brightness is constant, which is a consequence of the fact that Eq. (7) is true also in the case of nonzero energy spread and that the flux is also constant at zero detuning by symmetry. On the other hand, both the average brightness and degree of coherence, linked by Eq. (6), decrease significantly as a function of the energy spread. These results are in line with those of Ref. [4], which found that energy spread has no effect on the peak brightness but does degrade the coherence off axis.

We now turn to the source size and divergence. Figure 8 shows the 3D-projected distributions at various values of energy spread. As the energy spread increases, the radiation source divergence increases significantly. The effect on the spatial distribution is, however, more complex; the central
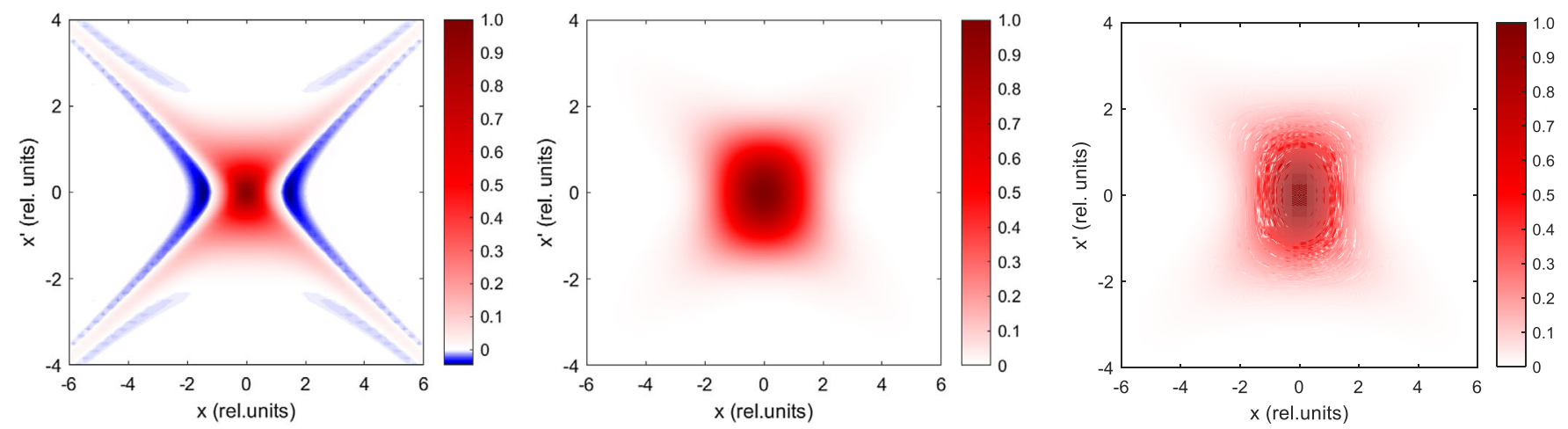

FIG. 6. Brightness distributions. Left: $\sigma_{E}=0.001, \varepsilon_{x}=\varepsilon_{y}=0$; middle: $\sigma_{E}=0, \varepsilon_{x}=\varepsilon_{y}=10$ pm, $\beta_{x}=\beta_{y}=2$; right: $\sigma_{E}=0.001$, $\varepsilon_{x}=\varepsilon_{y}=10 \mathrm{pm}, \beta_{x}=\beta_{y}=2$; normalized to unity in each case. 


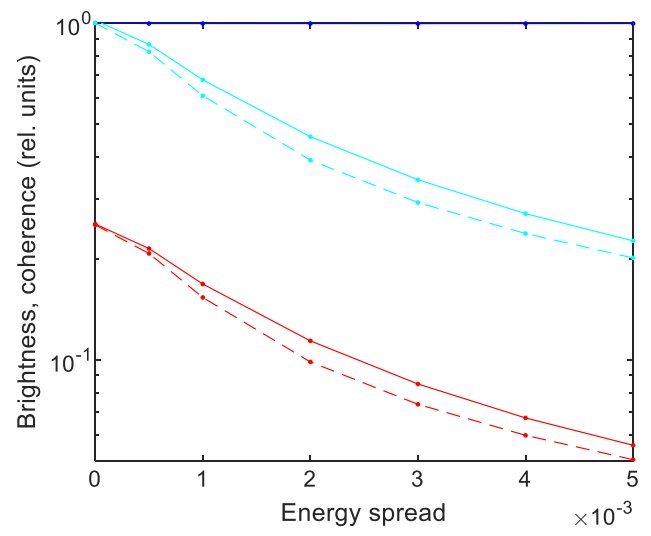

FIG. 7. Peak brightness (blue line), average brightness (red line), and degree of coherence (cyan line) as a function of the energy spread, at zero detuning; brightness values are normalized to the peak brightness at zero energy spread. Dashed curves: Gaussian approximation using Eqs. (14) and (15).

part of the distribution narrows, while the tails become increasingly wide, causing difficulties in determining the effective rms values.

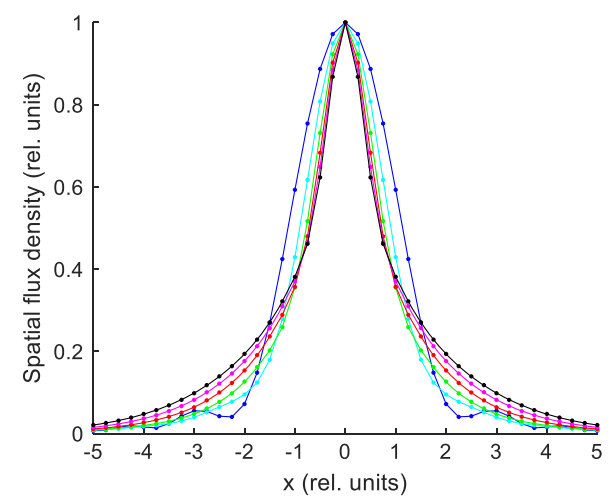

Figure 9 shows various estimates of the phase-space widths as a function of the energy spread. We also show (black dashed lines) the factors $Q_{a}$ and $Q_{s}$ for the increase in angular and spatial width, respectively, compared to the single-electron values derived in Ref. [19]:

$$
\sigma_{R^{\prime}}=Q_{a} \sigma_{R_{0}^{\prime}} \quad \text { and } \quad \sigma_{R}=Q_{s} \sigma_{R_{0}}
$$

with

$Q_{a}=\left[\frac{2 x^{2}}{-1+\exp \left(-2 x^{2}\right)+(2 \pi)^{1 / 2} \operatorname{erf}\left(2^{1 / 2} x\right)}\right]^{1 / 2}$ and

$Q_{s}=\left[Q_{a}(x / 4)\right]^{2 / 3}$,

where $x=2 \pi n N \sigma_{E}, \sigma_{R_{0}^{\prime}}=\sqrt{\lambda / 2 L}$, and (for convenience having removed a factor of 2 in the expression for $Q_{s}$ from that of Ref. [19]) $\sigma_{R_{0}}=(\lambda / 2 \pi) / \sigma_{R_{0}^{\prime}}$.

For the angular plane (Fig. 9, right), the core rms value (blue line) remains constant at about $\sim 0.5$ (in relative units), while the projected values (red and green lines)

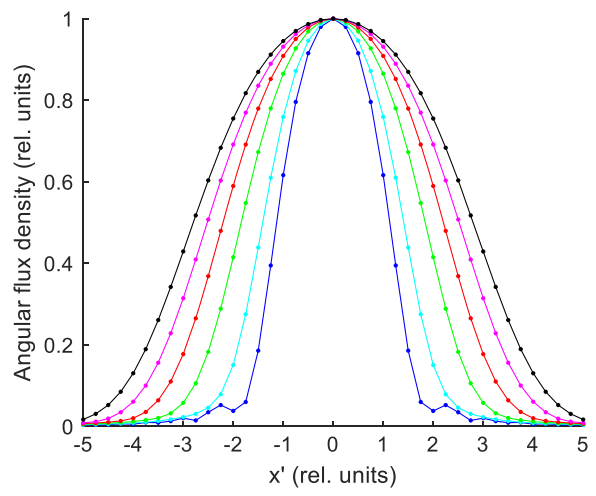

FIG. 8. 3D projected distributions along the $x$ (left) and $x^{\prime}$ (right) axes for rms energy spread varying between zero (blue line) and 0.005 (black line) in steps of 0.001 .
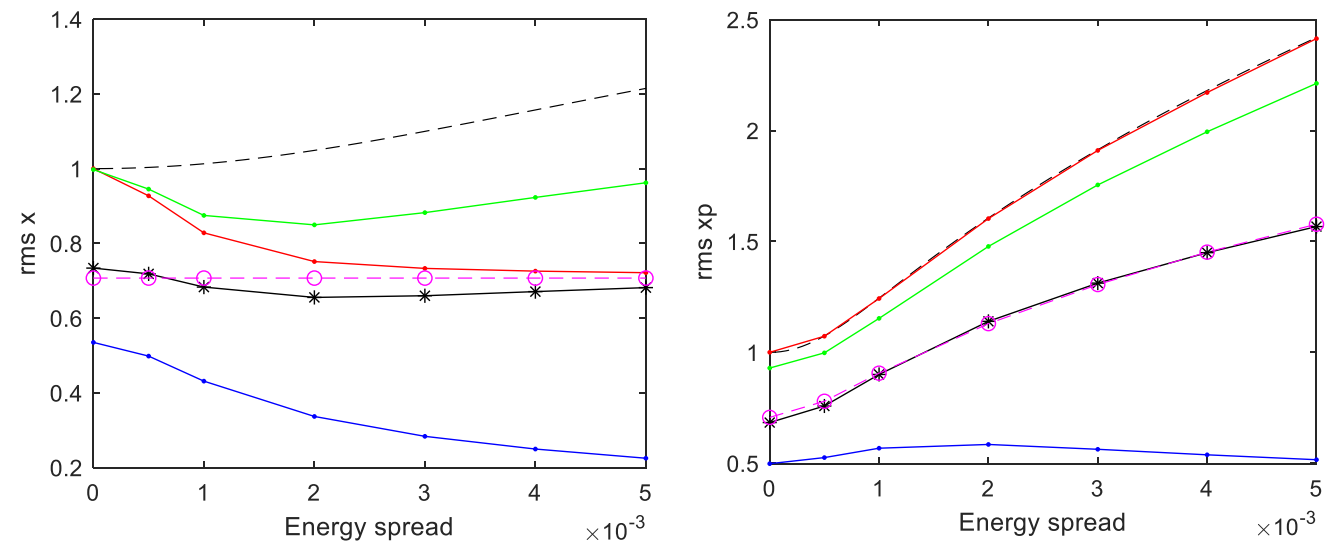

FIG. 9. rms beam sizes (left) and divergences (right) in relative units as a function of the energy spread obtained from 1D sections (blue line), 2D projections (red line), and full 3D projections (green line). Dashed black curve, from Eq. (12); black asterisks, fitted values from Eq. (13); magenta circles, Eq. (14). 
increase significantly. The 2D-projection rms values (red line) coincides with the function $Q_{a}$, as expected, since they both use the same method of determining the rms value. The 3D-projection data (green line) gives a slightly different result. As regards the spatial dimension (Fig. 9, left), all rms values first decrease (as expected from Fig. 8) and then the various quantities diverge. The function $Q_{s}$ differs significantly from these rms values and does not show the reduction in spatial dimension as the energy spread first increases.

We now examine the effective sizes and divergences that match the Gaussian approximation. Evidently, we cannot use Eq. (1), since $\mathcal{B}_{o}$ is independent of the energy spread, and so we use Eq. (3) to define

$$
\left(\sigma_{R} \sigma_{R^{\prime}}\right)_{\mathrm{fit}}=\frac{\lambda / 4 \pi}{\sqrt{\zeta}} .
$$

Figure 9 incudes the rms values derived from Eq. (13) and using the beta value $\left(\sigma_{R} / \sigma_{R^{\prime}}\right)$ derived from the $3 \mathrm{D}$-projected distributions. The values obtained in this way again lie in between the core and projected widths and can be reasonably well approximated by the following, shown as the dashed magenta lines in Fig. 9:

$$
\sigma_{R} \simeq \frac{\sigma_{R_{0}}}{\sqrt{2}}, \quad \sigma_{R^{\prime}} \simeq \frac{\sigma_{R_{0}^{\prime}}}{\sqrt{2}}\left(1+1.41 x^{2}\right)^{0.19} .
$$

The expression for the effective divergence has been obtained by a numerical fit, whereas the effective size is constant to within $10 \%$ and so has not been fitted. Figure 7 shows that using these values in Eq. (3) gives a good approximation to calculated values of the degree of coherence, within $\sim 12 \%$. Similarly, it follows from Eq. (6) that the average brightness is equally well approximated by

$$
\mathcal{B}_{\mathrm{av}}=\frac{F / 4}{4 \pi^{2} \Sigma_{x} \Sigma_{x^{\prime}} \Sigma_{y} \Sigma_{y^{\prime}}}
$$

Figure 10 shows that the radiation beta value is reduced significantly due to the energy spread, and this can be reasonably well approximated by the following (to within $10 \%)$, which follows from Eq. (14):

$$
\beta_{R}=\frac{\sigma_{R}}{\sigma_{R^{\prime}}} \simeq \frac{L / \pi}{\left(1+1.41 x^{2}\right)^{0.19}} .
$$

In conclusion, energy spread gives rise to a significant increase in the projected angular width and has a complicated effect on the spatial distribution, narrowing the core and introducing wide tails. Despite these effects, the peak brightness is not changed. This apparent contradiction is simply the result of the fact that the projected phase-space widths, as shown earlier, are not a good guide to the effect on peak brightness. On the other hand, the average brightness, and hence also the total degree of coherence, are significantly reduced by the energy spread, and Eq. (14)

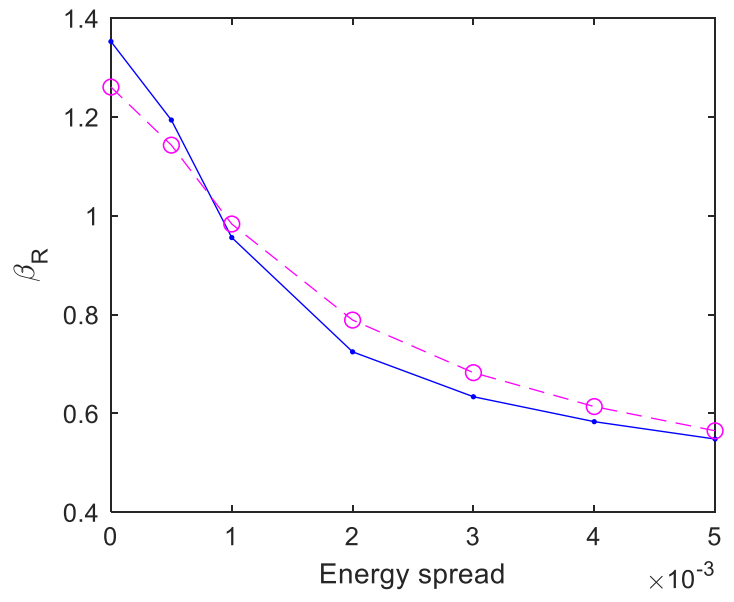

FIG. 10. Radiation beta value $\left(\beta_{R}\right)$ obtained from 3D projections as a function of the rms energy spread; blue line, calculated values; magenta line, Eq. (16).

presents a simple empirical model for the effective radiation source parameters that matches this using the Gaussian approximation.

\section{EFFECT OF ELECTRON BEAM EMITTANCE}

In order to include the effect of electron beam emittance, we make use of the "addition theorem" introduced by Kim [6] and convolute the brightness function with the electron beam density distribution. Assuming uncorrelated Gaussian distributions for the electron beam in each phasespace coordinate, the brightness can therefore be written as follows:

$$
\begin{aligned}
& \mathcal{B}\left(x, x^{\prime}, y, y^{\prime}\right) \\
& =\iiint \int \mathcal{B}\left(x-x_{o}, x^{\prime}-x_{o}^{\prime}, y-y_{o}, y^{\prime}-y_{o}^{\prime}\right) \\
& \quad \times P\left(x_{o}\right) P\left(x_{o}^{\prime}\right) P\left(y_{o}\right) P\left(y_{o}^{\prime}\right) d x_{o} d x_{o}^{\prime} d y_{o} d y_{o}^{\prime},
\end{aligned}
$$

where $P(z)=\exp -\left(z^{2} / 2 \sigma_{z}^{2}\right) / \sqrt{2 \pi} \sigma_{z}$, for $z=x, x^{\prime}, y, y^{\prime}$. Despite the conceptual simplicity, this is very demanding of computing time, especially if as here one wants to calculate the full 4D brightness distribution and, hence, the overall degree of coherence. Figure 6 (middle) shows the significant effect that a relatively small emittance of $10 \mathrm{pm}$ has on the brightness distribution.

Figure 11 shows the various measures of rms size and divergence as a function of the electron emittance. Also shown are the fitted values that result from Eq. (13), with the beta value determined from the projected distributions. At zero emittance, these values start with intermediate values between the core and projected widths, but as the emittance increases, they approach the projected values. The Gaussian approximation is correct as expected at zero emittance but then underestimates the effective size and 

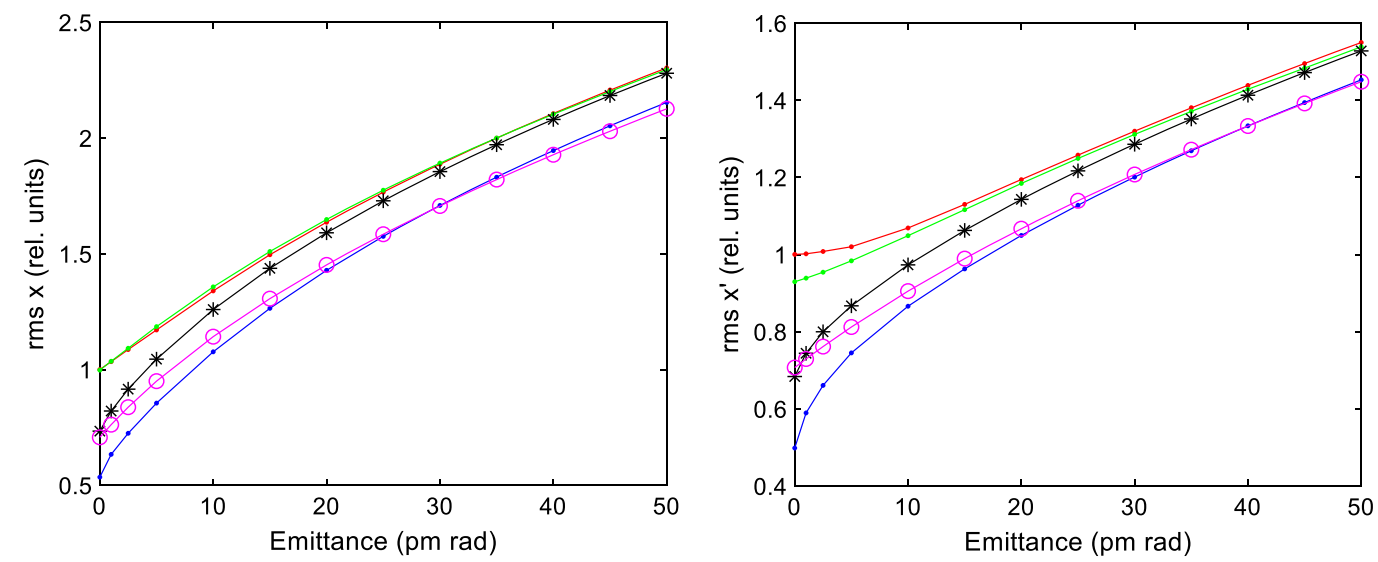

FIG. 11. rms beam sizes (left) and divergences (right) in relative units as a function of the emittance, with $\varepsilon_{x}=\varepsilon_{y}, \beta_{x}=\beta_{y}=2$, obtained from 1D sections (blue line), 2D projections (red line), and full 3D projections (green line); black asterisks, fitted values from Eq. (13); magenta, Gaussian approximation.
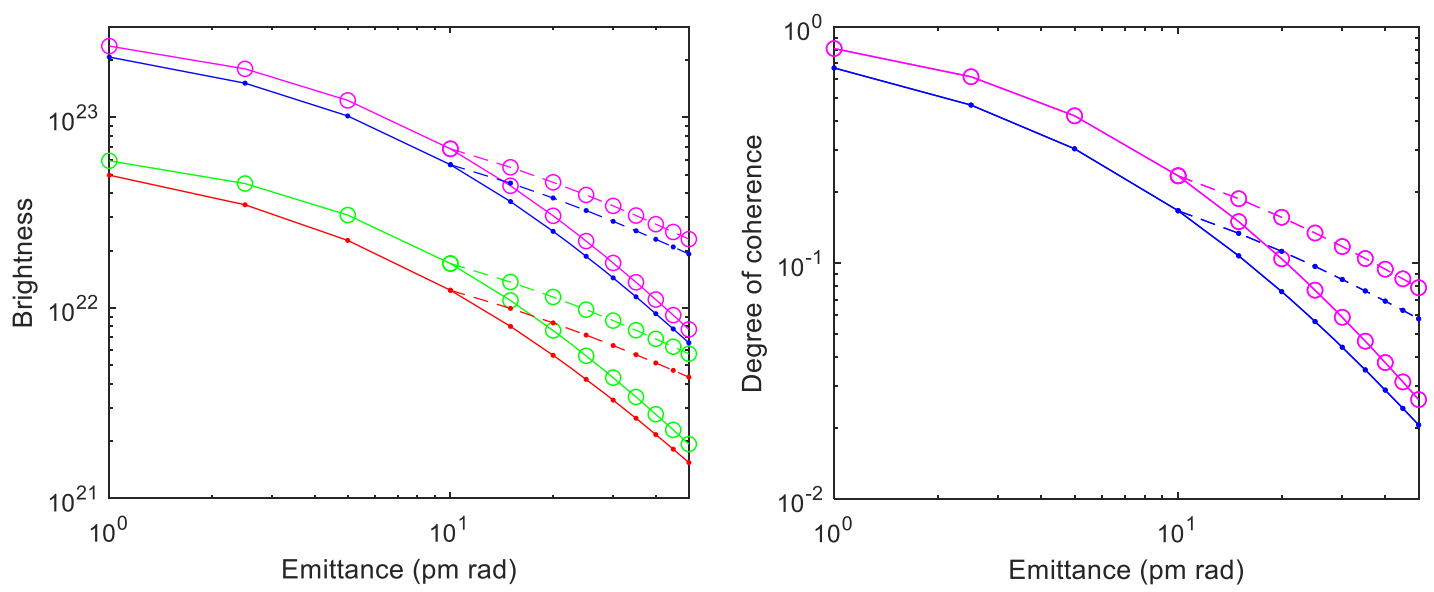

FIG. 12. Left: Peak brightness (blue) and average brightness (red); right: degree of coherence (blue) as a function of the emittance, with $\varepsilon_{x}=\varepsilon_{y}$ (solid lines) and $\varepsilon_{y}=10 \mathrm{pm}$ (dashed lines), both with $\beta_{x}=\beta_{y}=2$; magenta, Eqs. (1) and (3); green, Eq. (15).

divergence as the emittance increases. Nevertheless, as the emittance increases, all of the curves get relatively closer together, since in the limit of large emittance they must converge, and the maximum error in the Gaussian approximation is $10 \%$.

Figure 12 shows the peak brightness, average brightness, and coherence as a function of the electron beam emittance with zero energy spread. Solid lines are for equal emittances in both planes, and dashed lines are for a fixed vertical emittance of $10 \mathrm{pm}$ (for $\varepsilon_{x} \geq 10 \mathrm{pm}$ ). Also shown are the results obtained with the Gaussian approximation. It can be seen that the approximations follow very closely the trend of the calculated results, both when the emittance is equal in both planes as well as when they differ. The error in the GA is smallest at the low emittance and high emittance extremes, with errors of up to $40 \%$ in between. The fact that the approximations work well for both the peak and average brightness is a consequence of Eq. (8), which is valid both at zero emittance and large emittance, and remains roughly true (within 15\%) at intermediate values.

\section{COMBINED EFFECT OF ELECTRON BEAM EMITTANCE AND ENERGY SPREAD}

We now combine the effects of energy spread and emittance, by first incorporating energy spread according to Eq. (11) and then performing the convolution Eq. (17) for different emittances. Figure 6 (right) shows the small but noticeable additional effect on the brightness distribution of $0.1 \%$ energy spread when combined with an emittance of $10 \mathrm{pm}$.

Figure 13 shows the peak and average brightness, and degree of coherence, as a function of the emittance for various values of energy spread. Also shown is the Gaussian approximation. Above an emittance of $\sim 10 \mathrm{pm}$, all of the curves follow a similar trend, with decreasing relative gains 

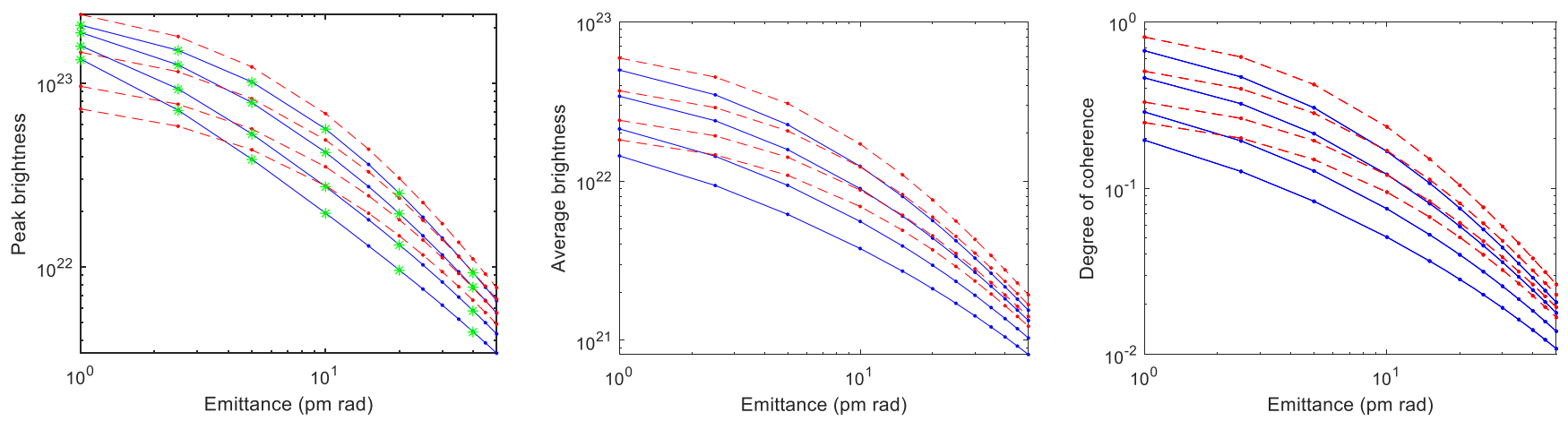

FIG. 13. Peak brightness (left), average brightness (middle), and degree of coherence (right) as a function of the emittance with $\varepsilon_{x}=\varepsilon_{y}, \beta_{x}=\beta_{y}=2$, for various values of the rms energy spread, 0.0 (uppermost curves), 0.001, 0.002, and 0.003 (lowest curves); blue, numerical results; red, Gaussian approximation; green asterisks, peak brightness values obtained with SPECTRA [20].

in brightness and coherence for a given reduction in emittance as the diffraction limit is approached. Below that point, the calculated peak brightness curves, which agree with those given by the well-known code SPECTRA [20], begin to deviate and tend to the same value independent of energy spread as seen in Sec. V.

The GA curves, which are, of course, identical in shape for all three quantities, follow the trend of the data reasonably well, the exception being the agreement with peak brightness for a nonzero energy spread and emittance less than about $10 \mathrm{pm}$, as noted above. The accuracy of the GA is actually slightly worse for average brightness and coherence than for peak brightness (above $10 \mathrm{pm}$ ) and gets worse with an increasing energy spread. For an energy spread up to $0.3 \%$, there is a maximum error of less than a factor of 2.

The relative effect of energy spread as a function of the emittance is shown in more detail in Fig. 14. It can be seen that the effect of the energy spread on the peak brightness and coherence increases as the emittance decreases, but

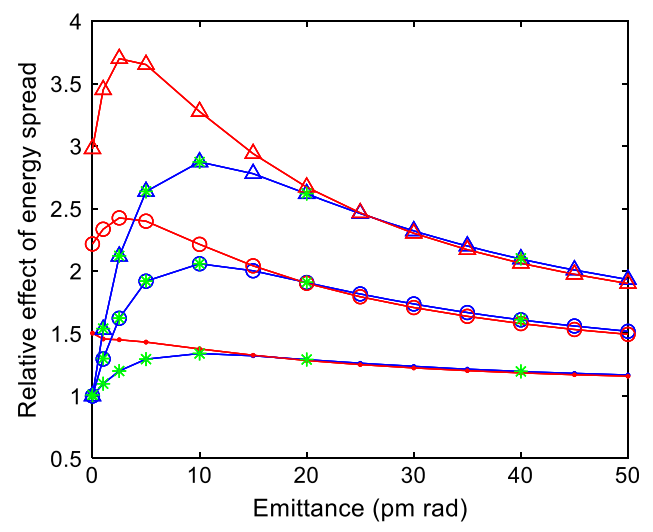

FIG. 14. Relative effect of energy spread on the peak brightness (blue) and degree of coherence (red) as a function of the emittance $\left(\varepsilon_{x}=\varepsilon_{y}, \beta_{x}=\beta_{y}=2\right)$; dots, $0.1 \%$; circles, $0.2 \%$; triangles, $0.3 \%$; green asterisks, peak brightness values obtained with SPECTRA [20]. below an emittance of $\sim 10-20 \mathrm{pm}$ the behavior of the two quantities diverges. The relative effect on the peak brightness decreases towards unity at zero emittance (i.e., no effect), as shown above, whereas the effect on the coherence continues to increase before decreasing slightly at a very small emittance, depending on the value of the energy spread.

The good agreement of the brightness and degree of coherence curves at a larger emittance suggests that these quantities are closely related. Figure 15 explores this in more detail by plotting the ratio between the degree of coherence $(\zeta)$ and an approximation for it based on the peak brightness:

$$
\zeta_{\text {approx }}=\frac{\mathcal{B}_{o}}{\left.\mathcal{B}_{o}\right|_{\varepsilon_{x}=\varepsilon_{y}=\sigma_{E}=0}} .
$$

It can be seen that in this example, provided the emittance is not too small, Eq. (18) gives a good estimate of the degree of coherence, to within about $10 \%$, which

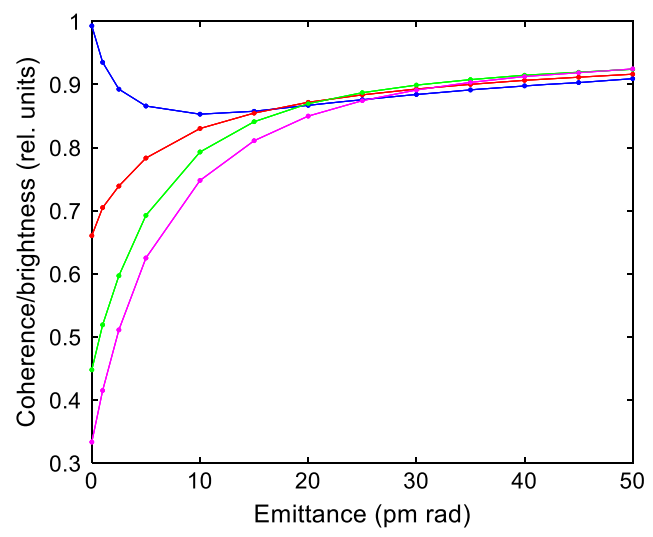

FIG. 15. Ratio of the degree of coherence to the approximation Eq. (18) as a function of the emittance ( $\left.\varepsilon_{x}=\varepsilon_{y}, \beta_{x}=\beta_{y}=2\right)$, for rms energy spread 0 (blue line), $0.1 \%$ (red line), $0.2 \%$ (green line), and $0.3 \%$ (magenta line). 

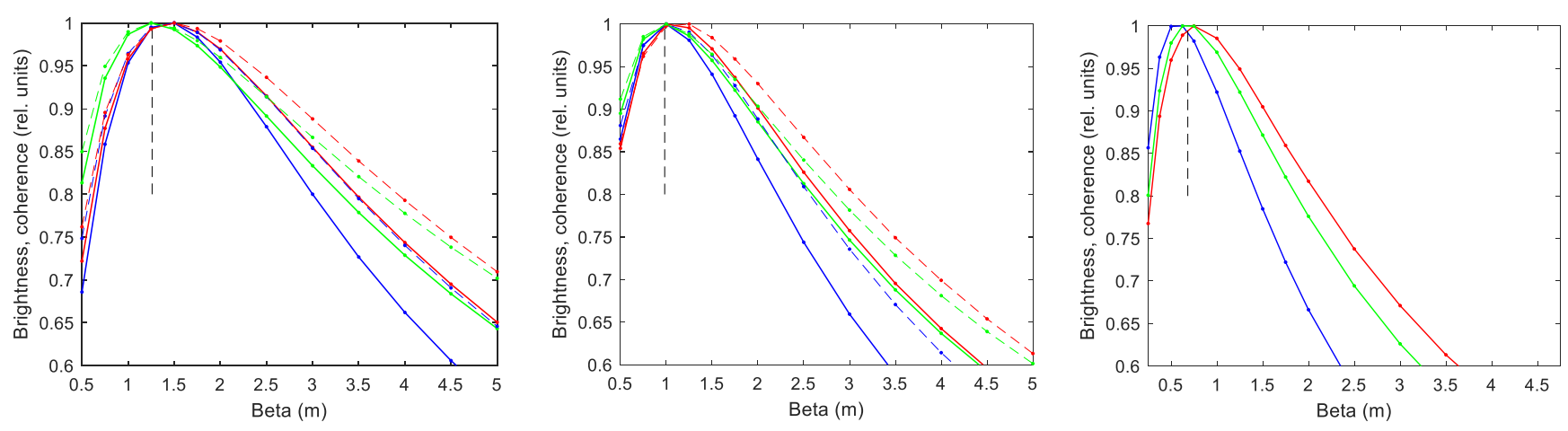

FIG. 16. Relative variation in the peak brightness (blue line) and degree of coherence (red line) as a function of the electron beam beta function, for a rms energy spread of zero (left), $0.1 \%$ (middle), and $0.3 \%$ (right); solid lines, $\varepsilon_{x}=\varepsilon_{y}=10$ pm; dashed lines (left and middle plots only), $\varepsilon_{x}=50 \mathrm{pm}, \varepsilon_{y}=10 \mathrm{pm}$, with $\beta_{x}=\beta_{y}$; green line, Gaussian approximation; dashed vertical line, from Eq. (16).

thus provides a much simpler way of calculating the degree of coherence than the very time-consuming full calculation. It is also more accurate than the Gaussian approximation and avoids the complications of determining the appropriate radiation parameters that enter in the equations.

\section{PHASE-SPACE MATCHING}

It is well known that to optimize the brightness and coherence requires a proper matching of the electron beam phase space to that of the photons through a correct choice of the electron beam beta values [5]. The importance of this in the context of diffraction-limited storage rings has been highlighted recently [3].

Figure 16 shows the relative variation of brightness and degree of coherence with the electron beam beta function for various values of emittance and energy spread. The optimum beta value which maximizes brightness and coherence clearly decreases as the energy spread increases, as expected from Fig. 10, and is close to the value given by Eq. (16), which is the peak of the Gaussian approximation. At zero energy spread, there is a slight discrepancy; the maximum brightness and coherence occurs at a beta of $\sim 1.5 \mathrm{~m}$, whereas the GA peaks at $\beta_{x, y}=L / \pi=1.26 \mathrm{~m}$. However, this has only a minor effect on the values of the brightness and coherence. Overall, it can be seen that the GA serves as a good guide to the optimum beta value and also the sensitivity to the beta value, which clearly increases as the energy spread increases. The curves with the larger horizontal emittance are slightly broader than those for the low emittance in both planes, but still the figures emphasize that in this low emittance regime it is important to provide a relatively low beta function at the undulator in order to optimize the radiation brightness and coherence.

\section{CONCLUSIONS}

The effects of electron beam energy spread and emittance on the brightness and coherence of undulator radiation have been examined using both the Wigner formulation and the commonly used Gaussian approximation, taking a $6 \mathrm{GeV}$ low emittance, near diffraction-limited, $x$-ray source such as PETRA IV as an example. As Fig. 13 clearly shows, the diffraction limit is approached only slowly with reducing emittance. Even at the emittance which is widely considered to be needed to reach the diffraction limit, $\varepsilon=\lambda / 4 \pi$, which corresponds to $10 \mathrm{pm}$ for a photon energy of $10 \mathrm{keV}$, the overall degree of spectral coherence is only $17 \%$, and to reach $50 \%$ would require an emittance of $2.5 \mathrm{pm}$ in each plane, which is beyond currently conceived machines.

Energy spread further impacts the brightness and coherence, and in the regime of emittance that is currently of interest, the relative effect increases as the electron beam emittance is reduced. For example, in a machine with $10 \mathrm{pm}$ emittance (in both planes), a typical $0.1 \% \mathrm{rms}$ energy spread reduces the degree of coherence by a further factor of 1.4 from $17 \%$ to $12 \%$, for radiation in the fundamental of the undulator spectrum. While this is not a big effect, it must be recalled that the effect of energy spread depends also on the harmonic number of the radiation. Thus, for $0.3 \%$ energy spread, equivalent to $0.1 \%$ for the third harmonic of the undulator spectrum (at the same photon energy), the degree of coherence in this example reduces by a factor 3.3 to $5 \%$. Special attention must therefore be paid to energy spread in the case of low-emittance and low-energy machines employing high harmonics in the undulator spectrum.

The results presented here confirm the observation in Ref. [4] that energy spread does not influence the peak brightness at zero emittance. Reference [4] also finds that there is no degradation of coherence on axis, but there is a degradation off axis, which is consistent therefore with the effect observed here on the overall degree of coherence. However, the statement in Ref. [4] that "there is no 'energy spread dominated' regime: when the emittance decreases so does also the influence of the energy spread on the coherence properties and the brightness" is misleading. Apart from the distinction being made between on-axis and off-axis coherence, 
it is clear from Fig. 14 that, until one reaches a very low emittance, the effect of energy spread increases as the emittance decreases, for both the peak brightness and degree of coherence. Furthermore, at a very low emittance there clearly is an "energy-spread-dominated regime," at least as regards the overall degree of coherence. This raises an interesting question as to whether peak brightness is a useful figure of merit, as opposed to the overall degree of coherence (or average brightness), which deserves further study.

It has been shown that, even though the brightness distribution based on the Wigner function is far from Gaussian in form, a simple Gaussian approximation with appropriate values for the radiation terms shows similar trends to those of much more elaborate calculations and is accurate to within a factor of 2 over the parameter range that has been investigated here. It also gives a good guide to the electron beam beta functions that optimize brightness and coherence as well as the sensitivity to the beta values.

There has, however, been a great deal of uncertainty in the past as to which are the appropriate radiation sizes and divergences to use in the Gaussian approximation to give the correct brightness and degree of coherence. It has been shown here that these are not the projected widths, as many authors have assumed, but they are intermediate between those obtained from sections and projections of the brightness distribution. At zero energy spread, the relevant quantities are given by model 4 in Table I. It has also been shown that previously derived expressions for the effect of energy spread [19], which were based on projected intensity distributions, are therefore not the most appropriate for the estimation of brightness and coherence.

Another possibility for estimating the degree of coherence has been suggested, at least in the regime which is sufficiently far from the diffraction limit, based on the peak brightness [Eq. (18)]. This is much easier to evaluate than a full calculation and also avoids the complications of determining the appropriate radiation parameters to use in the Gaussian approximation.

[1] D. Einfeld, M. Plesko, and J. Schaper, First multi-bend achromat lattice consideration, J. Synchrotron Radiat. 21, 856 (2014).

[2] M. Eriksson, E. Al-Dmour, Å. Andersson, M. Johansson, S. C. Leemann, L. Malmgren, P. F. Tavares and S. Thorin, Commissioning of the MAX IV light source, in Proceedings of the 7th International Particle Accelerator Conference (IPAC2016), Busan, Korea, 2016 (JACoW, Geneva, Switzerland, 2016), p. 11.

[3] L. Liu and H. Wesfahl, Jr., Towards diffraction limited storage ring based light sources, in Proceedings of the 8th International Particle Accelerator Conference (IPAC2017), Copenhagen, Denmark, 2017, (JACoW, Geneva, Switzerland, 2017, p. 1203.

[4] G. Geloni, S. Serkez, R. Khubbutdinov, V. Kocharyan, and E. Saldin, Effects of energy spread on brightness and coherence of undulator sources, J. Synchrotron Radiat. 25, 1335 (2018).

[5] S. Krinsky, Undulators as sources of synchrotron radiation, IEEE Trans. Nucl. Sci. 30, 3078 (1983).

[6] K-J. Kim, Brightness, coherence and propagation characteristics of synchrotron radiation, Nucl. Instrum. Methods Phys. Res., Sect. A 246, 71 (1986) (also Report No. LBL20181, 1985); see also K-J. Kim, X-ray Data Booklet (2009), Sec. II.1, http://xdb.lbl.gov/.

[7] R. Coisson and R. P. Walker, Phase space distribution of brilliance of undulator sources, Proc. SPIE Int. Soc. Opt. Eng. 582, 24 (1986).

[8] K.-J. Kim, Brightness and coherence of radiation from undulators and high-gain free electron lasers, in Proceedings of the 1987 Particle Accelerator Conference (IEEE, Washington, DC, 1987), p. 194.

[9] R. P. Walker, Insertion Devices: Undulators and Wigglers, in Proceedings of the CERN Accelerator School on Synchrotron Radiation and Free Electron Lasers, Grenoble, 1996 (CERN, Geneva, 1998), p. 129.

[10] P. Elleaume, in Undulators, Wigglers, and Their Applications, edited by H. Onuki and P. Elleaume (Taylor \& Francis, London, 2003), Chap. 3.

[11] R. R. Lindberg and K-J. Kim, Compact representations of partially coherent undulator radiation suitable for wave propagation, Phys. Rev. ST Accel. Beams 18, 090702 (2015).

[12] I. V. Bazarov, Synchrotron radiation representation in phase space, Phys. Rev. ST Accel. Beams 15, 050703 (2012).

[13] T. Tanaka, Numerical methods for characterization of synchrotron radiation based on the Wigner function method, Phys. Rev. ST Accel. Beams 17, 060702 (2014).

[14] G. Geloni, V. Kocharyan, and E. Saldin, Brightness of synchrotron radiation from undulators and bending magnets, J. Synchrotron Radiat. 22, 288 (2015).

[15] R. P. Walker, Interference effects in undulator and wiggler radiation sources, Nucl. Instrum. Methods Phys. Res., Sect. A 335, 328 (1993).

[16] M. Tischer (private communication).

[17] C. G. Schroer et al., PETRA IV: the ultralow-emittance source project at DESY, J. Synchrotron Radiat. 25, 1277 (2018).

[18] R. Coisson, Effective phase space widths of undulator radiation, Opt. Eng. 27, 250 (1988).

[19] T. Tanaka and H. Kitamura, Universal function for the brilliance of undulator radiation considering the energy spread effect, J. Synchrotron Radiat. 16, 380 (2009).

[20] T. Tanaka and H. Kitamura, SPECTRA: a synchrotron radiation calculation code, J. Synchrotron Radiat. 8, 1221 (2001); SPECTRA is available from http://spectrax .org/spectra/index.html. 